\title{
VEGFR-2 Expression in Glioblastoma Multiforme Depends on Inflammatory Tumor Microenvironment
}

\author{
Jana Jaal, ${ }^{1,2}$ Marju Kase, ${ }^{1,3}$ Ave Minajeva, ${ }^{2}$ Mikk Saretok, ${ }^{2}$ \\ Aidi Adamson, ${ }^{2}$ Jelizaveta Junninen, ${ }^{2}$ Tõnis Metsaots, ${ }^{2}$ Tõnu Jõgi, ${ }^{1}$ \\ Madis Joonsalu, ${ }^{1}$ Markus Vardja, ${ }^{1}$ and Toomas Asser ${ }^{2,4}$ \\ ${ }^{1}$ Hematology and Oncology Clinic, Department of Radiotherapy and Oncological Therapy, Tartu University Hospital, \\ 51003 Tartu, Estonia \\ ${ }^{2}$ Faculty of Medicine, University of Tartu, 50411 Tartu, Estonia \\ ${ }^{3}$ East-Tallinn Central Hospital, Center of Oncology, 10138 Tallinn, Estonia \\ ${ }^{4}$ Neurology Clinic, Department of Neurosurgery, Tartu University Hospital, 51014 Tartu, Estonia
}

Correspondence should be addressed to Jana Jaal; jana.jaal@kliinikum.ee

Received 30 July 2015; Revised 15 November 2015; Accepted 16 November 2015

Academic Editor: Alexander J. Steven

Copyright (c) 2015 Jana Jaal et al. This is an open access article distributed under the Creative Commons Attribution License, which permits unrestricted use, distribution, and reproduction in any medium, provided the original work is properly cited.

\begin{abstract}
Glioblastoma multiforme (GBM) is one of the most angiogenic tumors. However, antiangiogenic therapy has not shown significant clinical efficacy. The aim of our study was to evaluate the impact of inflammatory tumor microenvironment on the expression of vascular endothelial growth factor receptor 2 (VEGFR-2). Surgically excised primary GBM tissues were histologically examined for overall extent of inflammation (score 1-3). After immunohistochemistry, the tissue expression of ICAM-1 (optical density), the number of VEGFR-2 positive (VEGFR-2+) blood vessels (per microscopic field), and the endothelial staining intensity of VEGFR2 (score $0-3$ ) were determined. In GBM, the extent of inflammation was $1.9 \pm 0.7$ (group mean \pm SD). Mean optical density of inflammatory mediator ICAM-1 was $57.0 \pm 27.1$ (pixel values). The number of VEGFR-2+ blood vessels and endothelial VEGFR2 staining intensity were $6.2 \pm 2.4$ and $1.2 \pm 0.8$, respectively. A positive association was found between endothelial VEGFR-2 staining intensity and the extent of inflammation $(p=0.005)$. Moreover, VEGFR-2 staining intensity correlated with the expression level of ICAM-1 ( $p=0.026)$. The expression of VEGFR-2, one of the main targets of antiangiogenic therapy, depends on GBM microenvironment. Higher endothelial VEGFR-2 levels were seen in the presence of more pronounced inflammation. Target dependence on inflammatory tumor microenvironment has to be taken into consideration when treatment approaches that block VEGFR-2 signaling are designed.
\end{abstract}

\section{Introduction}

Although considered as rare tumor entity, about 27700 new primary CNS cancers are diagnosed each year in Europe [1]. Out of these, glioblastoma multiforme (GBM) is the most aggressive and lethal type of a brain tumor in adults that accounts for approximately $20 \%$ of all malignant primary CNS tumors and $82 \%$ of high grade (WHO grades III and IV) gliomas $[2,3]$. The prognosis of GBM patients is extremely dismal since current standard treatment options result only in medial survival times of 14.6 months [4].

GBM is one of the most angiogenic tumors. Therefore, in recent years, the inhibition of tumor angiogenesis has been an extremely attractive and dominating experimental therapeutic strategy in neurooncology $[5,6]$. A number of anticancer drugs that block formation of new blood vessels through different molecular targets and patterns of action are currently in various stages of clinical development for both newly diagnosed and recurrent GBM [7]. However, first promising results of angiogenesis inhibitors, such as high radiological response rates and apparent clinical benefit, have been replaced by prevalent disappointment. Major limitations of antiangiogenic drugs used in GBM include the modest number of durable responses and the lack of cytotoxic antitumor activity and overall survival benefit [6]. 
The reasons of the lack of significant clinical efficacy of antiangiogenic drugs in GBM, however, are not fully elucidated [8].

In GBM, at least five mechanisms by which tumors achieve neovascularization have been described: vascular cooption, angiogenesis, vasculogenesis, vascular mimicry, and glioblastoma-endothelial cell transdifferentiation [9]. Out of these, angiogenesis and vasculogenesis have been most extensively studied and described. During angiogenesis, blood vessels arise from sprouting and proliferation of endothelial cells from preexisting vascular network, whereas in vasculogenesis, de novo blood vessels are formed through colonization of circulating bone-marrow-derived endothelial progenitor cells that are recruited to the tumor [9]. Both previously mentioned mechanisms of neovascularization are largely regulated via vascular endothelial growth factor (VEGF) and its receptor 2 (VEGFR-2) [10]. It has been shown that tumor microenvironment influences glioblastoma treatment outcome $[11,12]$. The aim of the current study was to evaluate the impact of tumor microenvironment, particularly inflammatory reaction, on the expression of VEGFR-2, one of the main targets of antiangiogenic drugs.

\section{Materials and Methods}

Study was approved by the Research Ethics Committee of the University of Tartu, Estonia.

Surgically excised primary GBM tissues $(n=42)$ were obtained after primary operation (prior to radiotherapy) from patients treated at Tartu University Hospital or North Estonian Medical Centre.

2.1. Histology. Haematoxylin and eosin stained sections ( $4 \mu \mathrm{m}$ thick) were used for primary diagnosis. The diagnosis of GBM was confirmed by 2 independent pathologists. Afterwards, the overall extent of inflammatory reaction was estimated in tumor tissue by experienced pathologist. This was based on typical visual appearance of inflammation, including presence of edema and inflammatory cell infiltration. For the evaluation, an arbitrary score ranging from 1 to 3 was applied ( $1=$ weak, $2=$ moderate, and $3=$ strong inflammatory reaction).

2.2. Immunohistochemistry (IHC). GBM tissues were immunohistochemically examined for ICAM-1 and VEGFR-2 expression. Sections were cut from archived paraffin blocks and stained according to standard immunohistochemistry protocol. For immunohistochemistry, primary antibodies against ICAM-1 (Santa Cruz Biotechnology, Inc., \#sc-8439, dilution 1:100) and VEGFR-2 (Santa Cruz Biotechnology, Inc., \#sc-6251, dilution 1:100) were applied. Diaminobenzidine was used as chromogen.

The evaluation of immunohistochemically stained slides was carried out in a blinded fashion. First, immunohistochemical expression of intercellular adhesion molecule 1 (ICAM-1) was assessed. For this, digital IHC image analysis was performed. IHC digital image analysis was carried out in 6 selected images from each slide by using the freeware program ImageJ. The brown-colored area, occupied by the immunohistochemical reaction, was selected by the color threshold filtering tool to subtract the hematoxylin-stained areas at the background. Then the images were converted to the greyscale and the optical density by the area method was measured in pixel values ranging from 0 to 255 . Value 0 represents the lightest shade of the color while 255 represents the darkest shade of the color in the image. Tissue expression of ICAM-1 was determined at a magnification of $\times 10$.

The evaluation and scoring of VEGFR-2 immunohistochemically stained slides were carried out by 2 independent researchers. For VEGFR-2 expression, two parameters were assessed. First, the number of VEGFR-2 positive (VEGFR$2+$ ) blood vessels per microscopic field was determined. Additionally, endothelial VEGFR-2 staining intensity was evaluated using an arbitrary score ranging from 0 to $3(0=$ no staining; 1 = weak, 2 = moderate, and $3=$ strong staining intensity). For individual values, both parameters were determined in 5 microscopic fields. These values were used to evaluate the correlation between the assessments of 2 independent researchers. Afterwards, the mean number of VEGFR$2+$ blood vessels and VEGFR-2 staining intensity in 10 microscopic fields $(2 \times 5$ fields) were calculated. All VEGFR- 2 parameters were determined in areas with vital tumor tissue (excluding necrotic areas) at a magnification of $\times 40$.

2.3. Statistical Analysis. The SPSS statistical software was used to calculate individual means, group mean, and standard deviation of the mean. Additionally, Pearson correlation analysis was utilized. A $p$ value $<0.05$ was regarded statistically significant.

\section{Results}

3.1. Histology and IHC. In individual GBM samples, the extent of inflammation varied, ranging from 1.0 to 3.0 and being in the whole group $1.9 \pm 0.7$ (mean \pm SD). Figure 1 represents GBM tissues with weak (a), moderate (b), and strong (c) visual inflammatory reaction.

Similarly, individual optical densities of ICAM-1 in GBM tissue varied, ranging from to 17.6 to 154.9 pixel values. Group mean optical density of ICAM-1 was $57.0 \pm 27.1$ (mean \pm SD). Figure 2 illustrates GBM tissues with weak (a), moderate (b), and strong (c) optical density of ICAM-1.

VEGFR-2 parameters were determined by 2 independent researchers whose results were in good accordance $(R=$ $0.8, p<0.0001)$. In GBM tissue sections, the number of VEGFR-2+ blood vessels per microscopic field and endothelial VEGFR-2 staining intensity were $6.2 \pm 2.4$ (mean $\pm \mathrm{SD}$; range $2.8-13.5$ ) and $1.2 \pm 0.8$ (mean $\pm \mathrm{SD}$; range $0.0-2.8$ ), respectively. Figure 3 illustrates GBM tissues with weak (a), moderate (b), and strong (c) expression level of VEGFR-2 in tumor blood vessels.

3.2. Correlation Analysis. The results of correlation analysis are described in Table 1. A positive association was found between the extent of visual inflammation and endothelial VEGFR-2 staining intensity $(p=0.005)$. Moreover, endothelial VEGFR-2 staining intensity correlated with the 


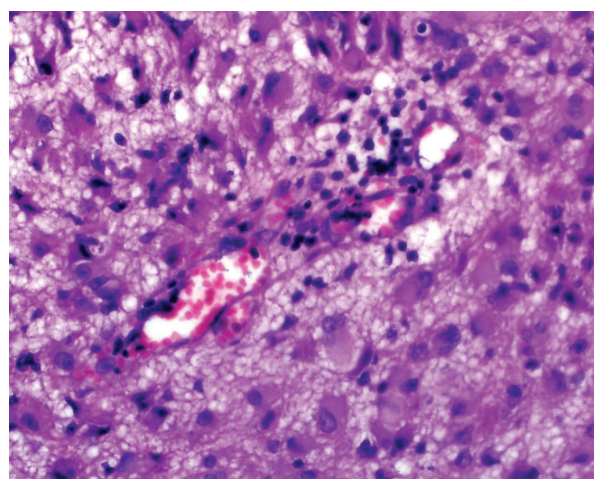

(a)

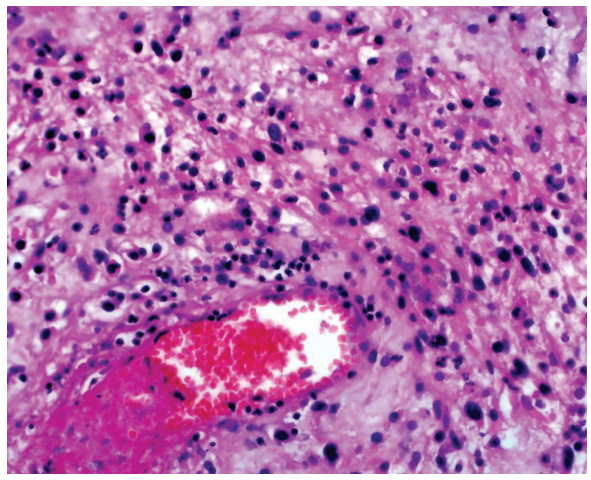

(b)

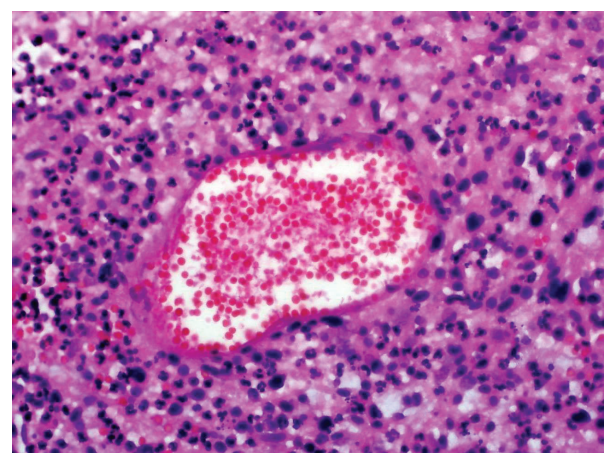

(c)

FIGURE 1: Inflammatory reaction in GBM. Photos illustrate GBM tissues with inflammatory reaction (photos represent different patients). (a) Weak (score 1) inflammation, (b) moderate (score 2) inflammation, and (c) strong (score 3) inflammation. Note the different numbers of tumor infiltrating inflammatory cells. Magnification $\times 40$.

TABLE 1: Results of correlation analysis*

\begin{tabular}{lc}
\hline Correlations & $p$ value \\
\hline $\begin{array}{l}\text { Visual inflammatory reaction and VEGFR-2 } \\
\text { staining intensity }\end{array}$ & $p=0.005$ \\
$\begin{array}{l}\text { Tissue ICAM-1 expression and VEGFR-2 staining } \\
\text { intensity }\end{array}$ & $p=0.026$ \\
$\begin{array}{l}\text { The number of VEGFR-2+ blood vessels and } \\
\text { VEGFR-2 staining intensity }\end{array}$ & $p=0.065$
\end{tabular}

${ }^{*}$ Bivariate Pearson correlation test.

expression level of tissue ICAM-1 $(p=0.026)$. Additionally, there was a trend toward significant association between the number of VEGFR-2 positive blood vessels and endothelial VEGFR-2 staining intensity in GBM tissue ( $p=0.065)$.

\section{Discussion}

Downstream effects of VEGFR-2 activation in the vascular endothelium include cell proliferation, migration, permeability, and survival, resulting in neovascularization processes, such as angiogenesis and vasculogenesis [10]. Consequently, this receptor has been very attractive target in the development of antiangiogenic drugs (e.g., bevacizumab, sunitinib, sorafenib, vatalanib, vandetanib, recentin, and cediranib)
[10]. Unfortunately, a number of these antiangiogenic drugs (vandetanib, cediranib, sorafenib, and sunitinib) have failed to show clinical efficacy in different phases of clinical trials in both newly diagnosed and recurrent glioblastoma [1317]. Moreover, the most advanced antiangiogenic drug in glioblastoma, bevacizumab, did not get approval from The European Medicines Agency Committee for Medicinal Products for Human Use (CHMP) due to the lack of clinically relevant efficacy $[18,19]$. All these negative trials have caused a lot of frustration since the results do not coincide with the initial expectations. The reasons of the lack of significant clinical efficacy of antiangiogenic drugs, however, are not fully elucidated.

In the present study, we evaluated the impact of tumor microenvironment on the expression level of VEGFR-2, one of the main targets of antiangiogenic drugs. Foremost, the possible role of inflammatory reaction was assessed. Inflammatory reaction was evaluated by two means. First, visual inflammation (based on the presence of tissue edema and inflammatory cell infiltration) was estimated in hematoxylineosin stained sections by experienced pathologist. Afterwards, to reduce subjectivity, a digital IHC image analysis was performed in ICAM-1 stained sections. ICAM-1 was chosen as a marker of inflammation since this transmembrane glycoprotein can be induced in response to a number of stimuli, including inflammatory mediators, hormones, 


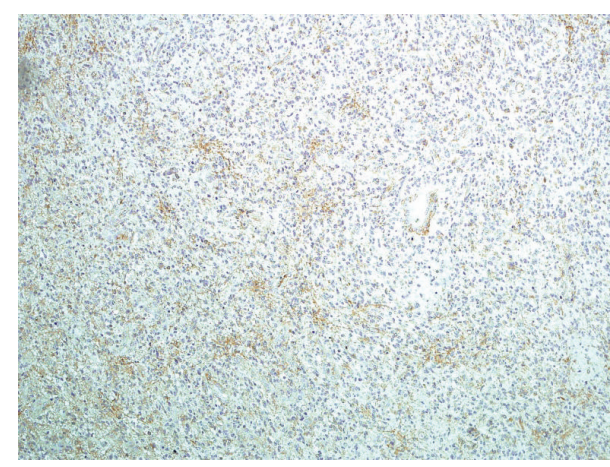

(a)

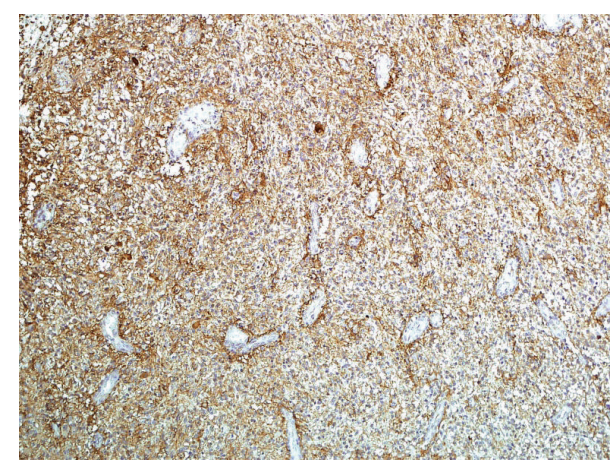

(b)

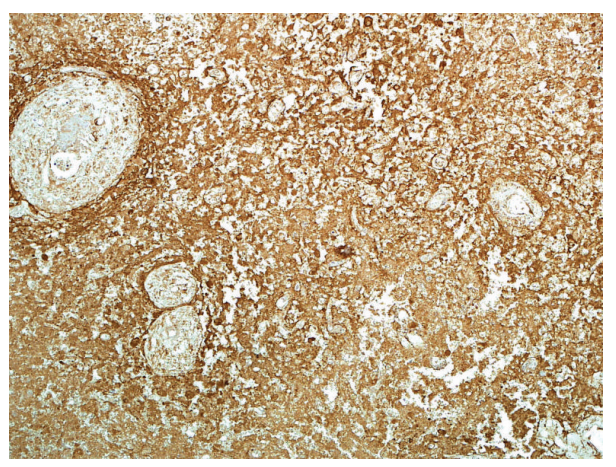

(c)

FIGURE 2: ICAM-1 expression in GBM. Photos illustrate GBM tissues with different extent of ICAM-1 expression (photos represent different patients). (a) Weak optical density, (b) moderate optical density, and (c) strong optical density. Magnification $\times 10$.

and cellular stresses [20, 21]. Moreover, endothelial ICAM1 is considered to represent the most important adhesion molecule for leukocyte recruitment to inflamed sites [22-24].

All glioblastoma samples showed various levels of visually confirmed inflammatory reaction. This is not surprising since inflammation is considered one of the characteristic histopathological features of glioblastoma [25]. Also, the expression of ICAM-1 was present in all digitally analyzed individual tumor samples, which is in good accordance with previous studies, where compared to peritumoral ICAM1 expression significantly higher expression of ICAM-1 has been detected in GBM tumor areas in both gene and protein levels [26-28]. In GBM cells, ICAM-1 expression has been shown to increase following stimulation with proinflammatory cytokines, such as interleukin-1 $\beta$ (IL-1 $\beta$ ), tumor necrosis factor-alpha (TNF $\alpha$ ), and interferon-gamma (IFN- $\gamma$ ) [26, 29], indicating that ICAM-1 is one of the inflammatory mediators also in this type of cancer.

In GBM tissues, different numbers of VEGFR-2+ blood vessels and endothelial levels of VEGFR-2 were detected. Previous studies have shown that in normal brain, low or undetectable endothelial expression of VEGFR-2 can be found; however, in gliomas, the proportion of VEGFR-2+ vessels and endothelial VEGFR-2 expression increases with tumor grade, being the highest in GBM $[30,31]$. Our study revealed that also in the most aggressive glioma-GBM-the extent of VEGFR-2 expression may vary. Additionally, present study showed that the expression of VEGFR-2 depends on inflammatory reaction in tumor tissue: the higher the endothelial VEGFR-2 expression the higher the extent of inflammation. Moreover, this association was seen for both assessments of inflammatory reaction (visual and computer software based).

Angiogenesis is a tightly controlled process that in a number of pathological conditions, including cancer and inflammation, may become aberrant [32]. Different factors produced by tissues are capable of promoting or inhibiting blood vessel proliferation, whereas in normal status, the balance between angiogenic and angiostatic factors exists. In inflammation, this balance is clearly inclined toward angiogenic factors and angiogenesis [33].

Although the link between inflammation and angiogenesis has received much attention only recently, there is a substantial body of evidence showing close association between these two processes. Previous studies have described that angiogenic factors exhibit both proangiogenic and proinflammatory effects, inflammatory cells are able to produce large quantities of proangiogenic factors, and both processes (inflammation and angiogenesis) are capable of potentiating each other [33]. For example, VEGF that exerts majority of its angiogenic effects by binding to VEGFR-2 has also been shown to induce adhesion molecules on endothelial cells during inflammation [34]. In endothelial cells, treatment with VEGF results in an increase of both ICAM-1 mRNA and protein expression [35]. Moreover, VEGF increases leukocyte 


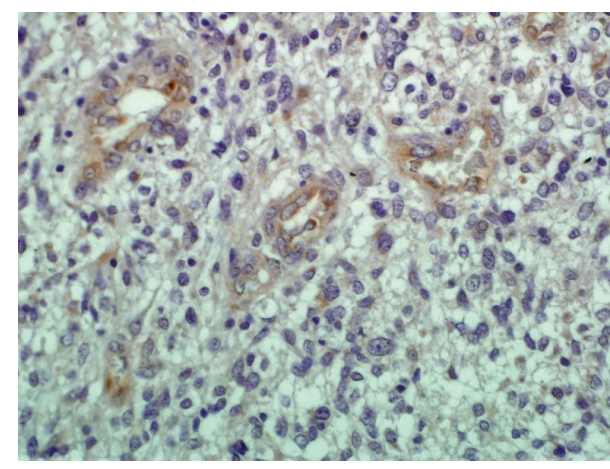

(a)

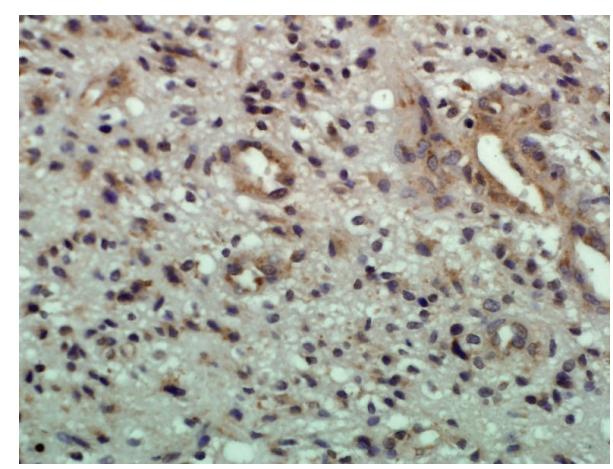

(b)

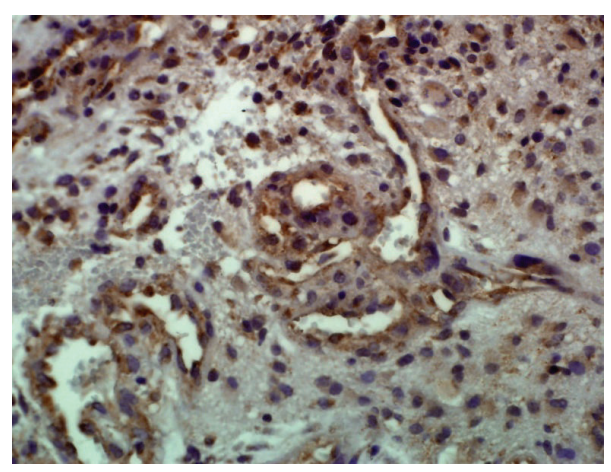

(c)

FIGURE 3: VEGFR-2 expression in GBM blood vessels. Photos illustrate GBM tissues with different endothelial staining intensities of VEGFR2 in blood vessels (photos represent different patients). (a) Weak (score 1) staining intensity, (b) moderate (score 2) staining intensity, and (c) strong (score 3) staining intensity. Note also the different numbers of VEGFR-2+ blood vessels. Magnification $\times 40$.

adhesiveness to endothelial cells, which is the first step of leukocyte trafficking into inflamed tissue [35]. Next to these effects, VEGF enhances vascular permeability and causes vasodilatation, potentiating thereby inflammation through formation of tissue edema $[10,36]$. At the same time, hyperpermeability is also involved in pathological angiogenesis [36]. Additionally, inflammatory and angiogenic processes involve similar cell types. Inflammatory cells, namely, monocytes, macrophages, T lymphocytes, and neutrophils, participate in the angiogenesis by secreting cytokines that affect endothelial cell functions, proliferation, migration, and activation [37]. Macrophages, present in the inflammatory infiltrate, produce a broad array of angiogenic growth factors and cytokines, generate channels for blood flow through proteolytic mechanisms, and promote the remodeling of arterioles into arteries [32]. Inflammatory dentritic cells stimulate similarly angiogenesis by secreting angiogenic factors and cytokines, as well as by promoting proangiogenic activity of T lymphocytes [32]. Previous studies have also shown that proinflammatory cytokines, which are always present in inflamed tissue, mediate also endothelial expression of VEGFR-2 [38, 39]. Later this is also indirectly supported by our findings since positive correlation was found between the extent of VEGFR-2 expression and inflammatory response in GBM tissue.

There are several clinical situations, where inflammatory reaction in GBM may be suppressed. These particularly include the use of anti-inflammatory drugs, such as steroids and nonsteroidal anti-inflammatory drugs (NSAIDs), to manage tumor surrounding inflammation and edema [40]. Whether these very commonly used medicines influence also treatment efficacy of antiangiogenic drugs through diminishing inflammatory response and thereby the expression of VEGFR-2 remains unclear. Nevertheless, our data point toward the possibility that this association might exist. This is also supported by studies where dexamethasone, most frequently used steroid in GBM patients, has been shown to inhibit the effects of proinflammatory cytokines, VEGF mRNA expression, VEGFR-2 expression, and macrophage infiltration $[39,41,42]$.

\section{Conclusions}

In conclusion, our study showed that the expression of VEGFR-2, one of the main targets of antiangiogenic drugs, depends on GBM microenvironment. Importantly, higher endothelial VEGFR-2 levels were seen in the presence of more pronounced inflammation, whereas in less inflamed tissues only weak expression of VEGFR-2 was found. Target dependence on inflammatory tumor microenvironment has to be taken into consideration when treatment approaches that block VEGFR-2 signaling are designed.

\section{Conflict of Interests}

The authors declare that there is no conflict of interests regarding the publication of this paper. 


\section{Acknowledgment}

This work was supported by Estonian Research Council (Grant no. IUT2-4).

\section{References}

[1] E. Crocetti, A. Trama, C. Stiller et al., "Epidemiology of glial and non-glial brain tumours in Europe," European Journal of Cancer, vol. 48, no. 10, pp. 1532-1542, 2012.

[2] T. A. Dolecek, J. M. Propp, N. E. Stroup, and C. Kruchko, "CBTRUS statistical report: primary brain and central nervous system tumors diagnosed in the United States in 2005-2009," Neuro-Oncology, vol. 14, supplement 5, pp. vl-v49, 2012.

[3] C.-H. Lee, K.-W. Jung, H. Yoo, S. Park, and S. H. Lee, "Epidemiology of primary brain and central nervous system tumors in Korea," Journal of Korean Neurosurgical Society, vol. 48, no. 2, pp. 145-152, 2010.

[4] R. Stupp, W. P. Mason, M. J. van den Bent et al., "Radiotherapy plus concomitant and adjuvant temozolomide for glioblastoma," The New England Journal of Medicine, vol. 352, no. 10, pp. 987-996, 2005.

[5] A. D. Norden, J. Drappatz, and P. Y. Wen, "Antiangiogenic therapies for high-grade glioma," Nature Reviews Neurology, vol. 5, no. 11, pp. 610-620, 2009.

[6] K. Seystahl and M. Weller, "Is there a world beyond bevacizumab in targeting angiogenesis in glioblastoma?" Expert Opinion on Investigational Drugs, vol. 21, no. 5, pp. 605-617, 2012.

[7] E. Timotheadou, "New agents targeting angiogenesis in glioblastoma," Chemotherapy Research and Practice, vol. 2011, Article ID 878912, 8 pages, 2011.

[8] R. Rahman, S. Smith, C. Rahman, and R. Grundy, "Antiangiogenic therapy and mechanisms of tumor resistance in malignant glioma," Journal of Oncology, vol. 2010, Article ID 251231, 16 pages, 2010.

[9] M. E. Hardee and D. Zagzag, "Mechanisms of glioma-associated neovascularization," The American Journal of Pathology, vol. 181, no. 4, pp. 1126-1141, 2012.

[10] K. Holmes, O. L. Roberts, A. M. Thomas, and M. J. Cross, "Vascular endothelial growth factor receptor-2: structure, function, intracellular signalling and therapeutic inhibition," Cellular Signalling, vol. 19, no. 10, pp. 2003-2012, 2007.

[11] L. A. D. Cooper, D. A. Gutman, C. Chisolm et al., "The tumor microenvironment strongly impacts master transcriptional regulators and gene expression class of glioblastoma," American Journal of Pathology, vol. 180, no. 5, pp. 2108-2119, 2012.

[12] M. Kase, A. Adamson, M. Saretok et al., "Impact of tumor infiltrating CD63 positive cells on survival in patients with glioblastoma multiforme," Journal of Neurosurgical Sciences, In press.

[13] T. N. Kreisl, K. A. McNeill, J. Sul, F. M. Iwamoto, J. Shih, and H. A. Fine, "A phase I/II trial of vandetanib for patients with recurrent malignant glioma," Neuro-Oncology, vol. 14, no. 12, pp. 1519-1526, 2012.

[14] T. T. Batchelor, P. Mulholland, B. Neyns et al., "Phase III randomized trial comparing the efficacy of cediranib as monotherapy, and in combination with lomustine, versus lomustine alone in patients with recurrent glioblastoma," Journal of Clinical Oncology, vol. 31, no. 26, pp. 3212-3218, 2013.
[15] J. D. Hainsworth, T. Ervin, E. Friedman et al., "Concurrent radiotherapy and temozolomide followed by temozolomide and sorafenib in the first-line treatment of patients with glioblastoma multiforme," Cancer, vol. 116, no. 15, pp. 3663-3669, 2010.

[16] E. Pan, D. Yu, B. Yue et al., "A prospective phase II singleinstitution trial of sunitinib for recurrent malignant glioma," Journal of Neuro-Oncology, vol. 110, no. 1, pp. 111-118, 2012.

[17] M. Hutterer, M. Nowosielski, J. Haybaeck et al., "A single-arm phase II Austrian/German multicenter trial on continuous daily sunitinib in primary glioblastoma at first recurrence (SURGE 01-07)," Neuro-Oncology, vol. 16, no. 1, pp. 92-102, 2014.

[18] M. R. Gilbert, J. J. Dignam, T. S. Armstrong et al., "A randomized trial of bevacizumab for newly diagnosed glioblastoma," The New England Journal of Medicine, vol. 370, no. 8, pp. 699-708, 2014.

[19] O. L. Chinot, W. Wick, W. Mason et al., "Bevacizumab plus radiotherapy-temozolomide for newly diagnosed glioblastoma," The New England Journal of Medicine, vol. 370, no. 8, pp. 709-722, 2014.

[20] K. A. Roebuck and A. Finnegan, "Regulation of intercellular adhesion molecule-1 (CD54) gene expression," Journal of Leukocyte Biology, vol. 66, no. 6, pp. 876-888, 1999.

[21] C.-C. Chen, "Signal transduction pathways of inflammatory gene expressions and therapeutic implications," Current Pharmaceutical Design, vol. 12, no. 27, pp. 3497-3508, 2006.

[22] G. A. Koning, R. M. Schiffelers, and G. Storm, "Endothelial cells at inflammatory sites as target for therapeutic intervention," Endothelium, vol. 9, no. 3, pp. 161-171, 2002.

[23] S. Hua, "Targeting sites of inflammation: intercellular adhesion molecule-1 as a target for novel inflammatory therapies," Frontiers in Pharmacology, vol. 4, article 127, 2013.

[24] A. K. Hubbard and R. Rothlein, "Intercellular adhesion molecule-1 (ICAM-1) expression and cell signaling cascades," Free Radical Biology and Medicine, vol. 28, no. 9, pp. 1379-1386, 2000.

[25] C. Piperi, M. S. Themistocleous, G. A. Papavassiliou et al., "High incidence of MGMT and RARbeta promoter methylation in primary glioblastomas: association with histopathological characteristics, inflammatory mediators and clinical outcome," Molecular Medicine, vol. 16, no. 1-2, pp. 1-9, 2010.

[26] M. C. Kuppner, E. van Meir, M. F. Hamou, and N. de Tribolet, "Cytokine regulation of intercellular adhesion molecule-1 (ICAM-1) expression on human glioblastoma cells," Clinical and Experimental Immunology, vol. 81, no. 1, pp. 142-148, 1990.

[27] I. Berindan-Neagoe, R. Chiorean, C. Braicu et al., "Quantitative mRNA expression of genes involved in angiogenesis, coagulation and inflammation in multiforme glioblastoma tumoral tissue versus peritumoral brain tissue: lack of correlation with clinical data," European Cytokine Network, vol. 23, no. 2, pp. 4555, 2012.

[28] M.-C. Gingras, E. Roussel, J. M. Bruner, C. D. Branch, and R. P. Moser, "Comparison of cell adhesion molecule expression between glioblastoma multiforme and autologous normal brain tissue," Journal of Neuroimmunology, vol. 57, no. 1-2, pp. 143-153, 1995.

[29] A. Meager, "Bioimmunoassays for proinflammatory cytokines involving cytokine-induced cellular adhesion molecule expression in human glioblastoma cell lines," Journal of Immunological Methods, vol. 190, no. 2, pp. 235-244, 1996.

[30] E. A. Kuczynski, S. G. Patten, and B. L. Coomber, "VEGFR2 expression and TGF- $\beta$ signaling in initial and recurrent highgrade human glioma," Oncology, vol. 81, no. 2, pp. 126-134, 2011. 
[31] H. Huang, J. Held-Feindt, R. Buhl, H. M. Mehdorn, and R. Mentlein, "Expression of VEGF and its receptors in different brain tumors," Neurological Research, vol. 27, no. 4, pp. 371-377, 2005.

[32] Z. M. Dong, A. C. Aplin, and R. F. Nicosia, "Regulation of angiogenesis by macrophages, dendritic cells, and circulating myelomonocytic cells," Current Pharmaceutical Design, vol. 15, no. 4, pp. 365-379, 2009.

[33] D. N. Granger and E. Senchenkova, "Angiogenesis," in Inflammation and the Microcirculation, chapter 6, Morgan \& Claypool Life Sciences, San Rafael, Calif, USA, 2010, http://www.ncbi .nlm.nih.gov/books/NBK53377/.

[34] M. Lohela, M. Bry, T. Tammela, and K. Alitalo, "VEGFs and receptors involved in angiogenesis versus lymphangiogenesis," Current Opinion in Cell Biology, vol. 21, no. 2, pp. 154-165, 2009.

[35] I. Kim, S.-O. Moon, S. H. Kim, H. J. Kim, Y. S. Koh, and G. Y. Koh, "Vascular endothelial growth factor expression of intercellular adhesion molecule 1 (ICAM-1), vascular cell adhesion molecule 1 (VCAM-1), and E-selectin through nuclear factor-kappa B activation in endothelial cells," The Journal of Biological Chemistry, vol. 276, no. 10, pp. 7614-7620, 2001.

[36] J. A. Nagy, L. Benjamin, H. Zeng, A. M. Dvorak, and H. F. Dvorak, "Vascular permeability, vascular hyperpermeability and angiogenesis," Angiogenesis, vol. 11, no. 2, pp. 109-119, 2008.

[37] A. Naldini and F. Carraro, "Role of inflammatory mediators in angiogenesis," Current Drug Targets: Inflammation and Allergy, vol. 4, no. 1, pp. 3-8, 2005.

[38] J. S. Yao, W. Zhai, W. L. Young, and G.-Y. Yang, "Interleukin-6 triggers human cerebral endothelial cells proliferation and migration: the role for KDR and MMP-9," Biochemical and Biophysical Research Communications, vol. 342, no. 4, pp. 13961404, 2006.

[39] A. Ristimäki, K. Narko, B. Enholm, V. Joukov, and K. Alitalo, "Proinflammatory cytokines regulate expression of the lymphatic endothelial mitogen vascular endothelial growth factorC," Journal of Biological Chemistry, vol. 273, no. 14, pp. 84138418, 1998.

[40] P. Roth, L. Regli, M. Tonder, and M. Weller, "Tumor-associated edema in brain cancer patients: pathogenesis and management," Expert Review of Anticancer Therapy, vol. 13, no. 11, pp. 13191325, 2013.

[41] K. Narko, B. Enholm, T. Mäkinen, and A. Ristimäki, "Effect of inflammatory cytokines on the expression of the vascular endothelial growth factor-C," International Journal of Experimental Pathology, vol. 80, no. 3, pp. 109-112, 1999.

[42] N. Zhang, Z. Fang, P. R. Contag, A. F. Purchio, and D. B. West, "Tracking angiogenesis induced by skin wounding and contact hypersensitivity using a Vegfr2-luciferase transgenic mouse," Blood, vol. 103, no. 2, pp. 617-626, 2004. 


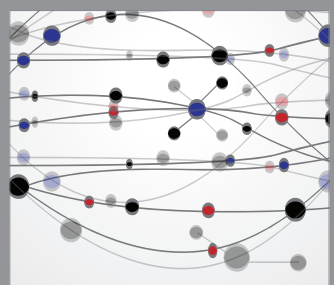

The Scientific World Journal
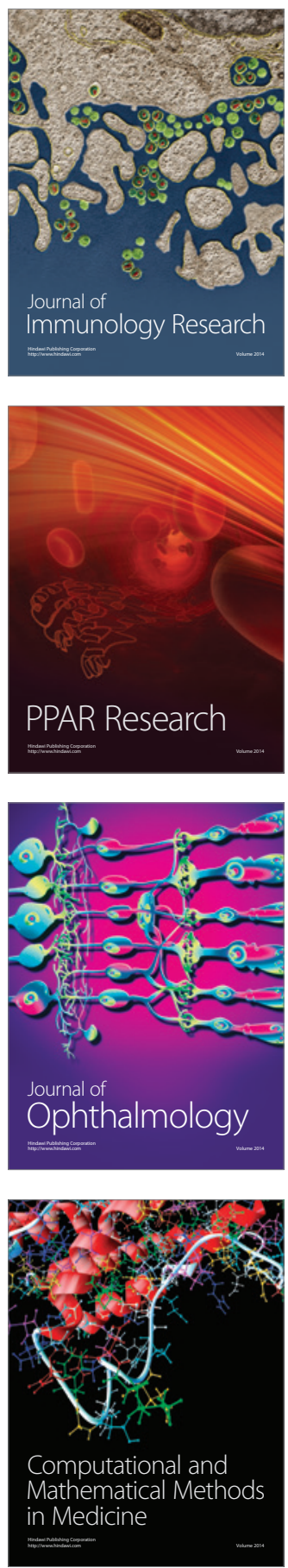

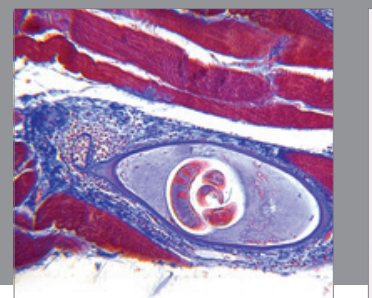

Gastroenterology

Research and Practice
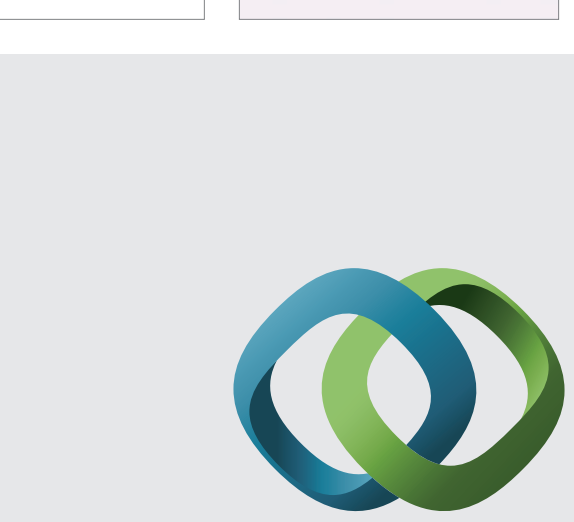

\section{Hindawi}

Submit your manuscripts at

http://www.hindawi.com
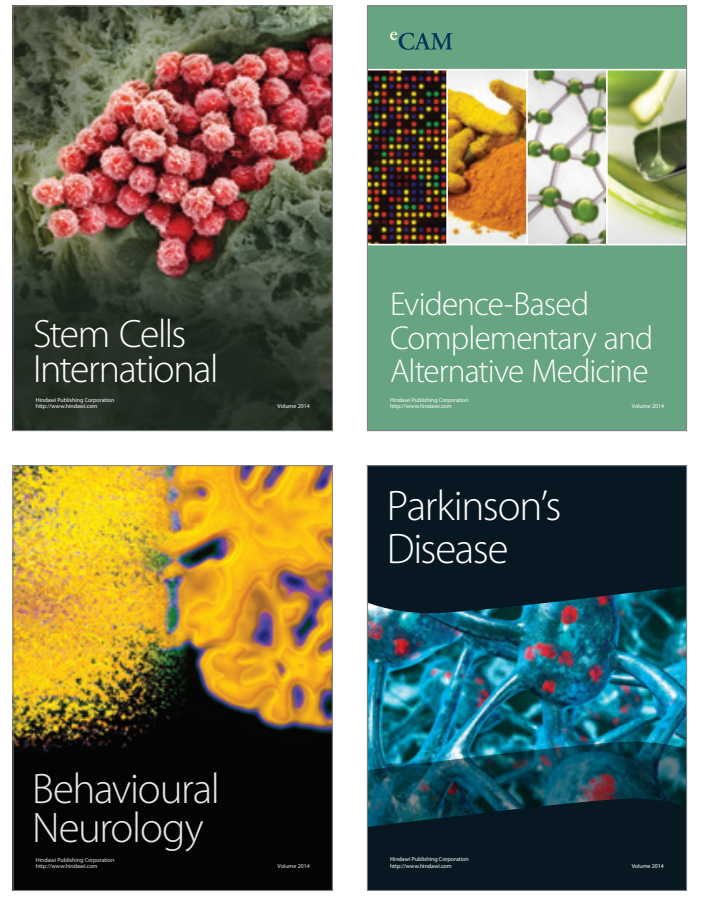
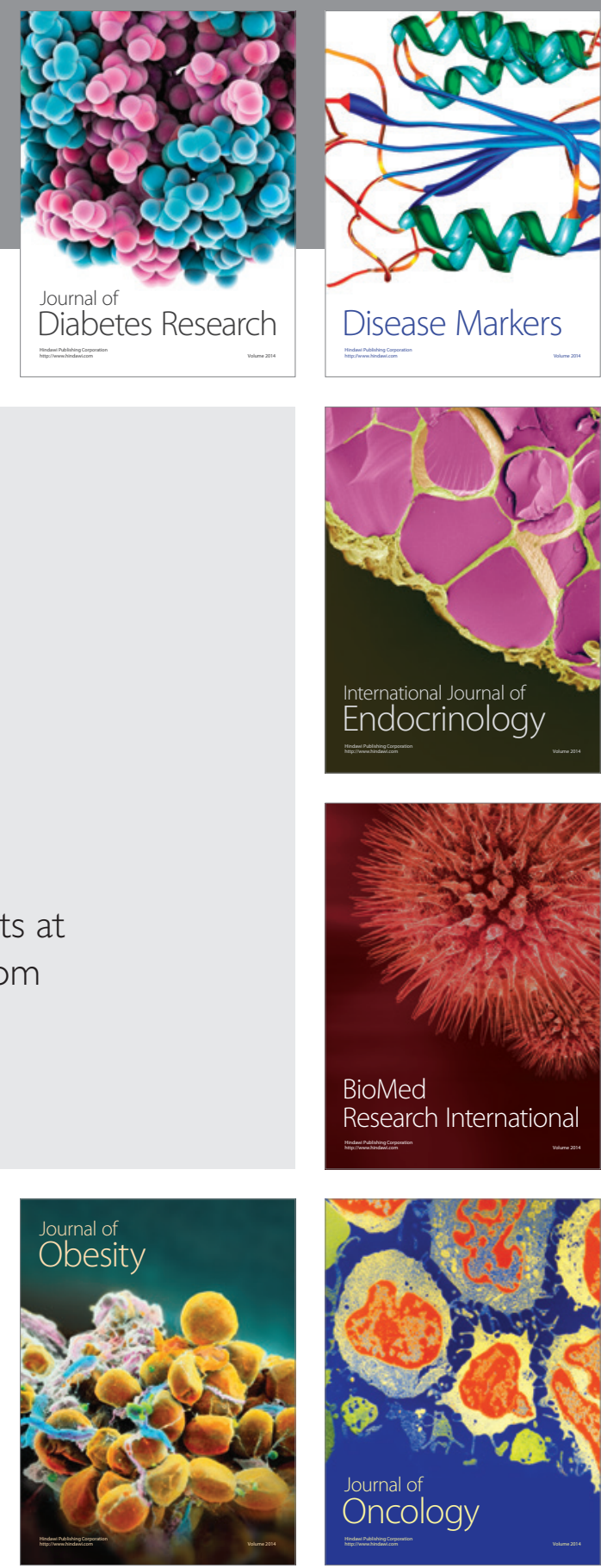

Disease Markers
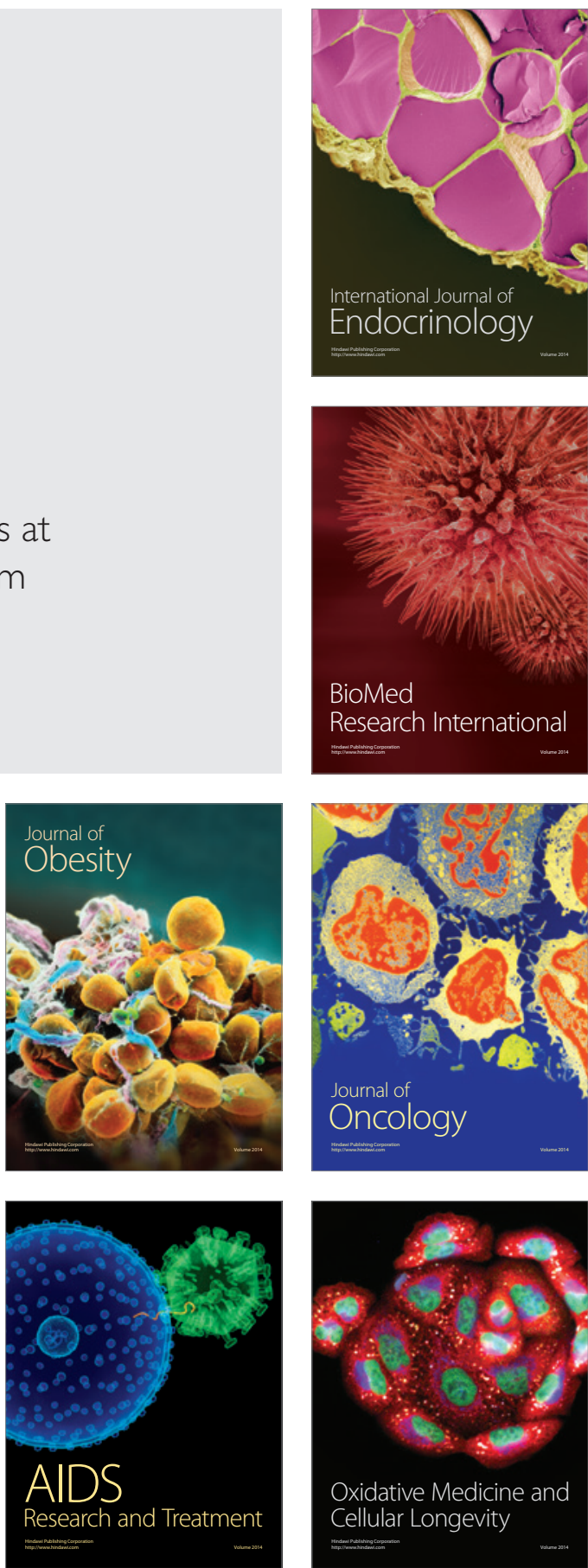